ent from one another. Suppose that to every circle c contained with its interior in the region $R$ corresponds a point set of $R^{\prime}$ which consists of all the points of a closed circle $c^{\prime}$. Then the point set $R^{\prime}$ is itself a region and the transformation of $R$ into $R^{\prime}$ is analytic and either a direct or an inverse transformation of Möbius.

It is not difficult to generalize this result by restricting the class of circles $c$ belonging to $R$ which are supposed to be transformed into circles. Take for instance a continuous positive function $\phi(P)$ defined everywhere in the region $R$. Then the theorem holds if we suppose that every circle of center $P$ and whose radius is less than $\phi(P)$ is transformed into a circle lying on $R^{\prime}$.

The following generalization of Theorem 2 is nearly self evident if we note that three circles in space cutting one another at six different points must lie on the same sphere.

THEOREm 3. Supposing that a plane region $R$ is transformed by $a$ one to one correspondence into an arbitrary point set $R^{\prime}$ lying in $n$-space $(n \geqq 3)$ under the same assumptions as before, then $R^{\prime}$ must be a two dimensional sphere or a plane and the transformation is a transformation of Möbius.

Other generalizations can also be imagined which, however, are outside the scope of this note.

UNIVERSITY OF MUNICH

\title{
A NOTE ON DETERMINANTS
}

BY M. H. INGRAHAM

The following theorem, with its corollary, when applicable affords great numerical simplifications. Although the writer has tried and failed to discover it in the literature, it is submitted with some hesitation lest it may not be new. The corollary was discovered and proved by the author. Upon seeing it Professor Max Zorn suggested the more general form of the theorem, which was then proved by the author.

Theorem. If $A$ is a square matrix $\left(A_{i j}\right),(i, j=1, \cdots, n)$, where the $A_{i j}$ are $m \times m$ matrices which are commutative in pairs, and if $B$ is the $m \times m$ matrix which is arrived at by taking the 
determinant of $A$ with the $A_{i j}$ as elements, then the determinant of $B$ is equal to the determinant of $A$.

Corollary. If $\left(G(x)=g_{i j}(x)\right)$ is a square matrix whose elements are polynomials $g_{i j}(x)$ and if $M$ is a square matrix of constant elements, then if the determinant of $G(x)$ is $g(x)$, the determinant of $G(M)$ (that is, the matrix of matrices $g_{i j}(M)$ ) is the determinant of $g(M)$.

The method of proof is by induction on $n$. We will first consider the case where the field to which the elements of the $A_{i j}$ belong has an infinite number of elements. It will be sufficient to show that the theorem holds when $A$ is replaced by $A-\lambda I$ and $A_{i i}$ by $A_{i i}-\lambda I$, where $A_{11}-\lambda I$ is non-singular, since the identity established will hold for an infinitude of values of $\lambda$ and hence for $\lambda=0$.

Consider $A^{(1)}=(A-\lambda I) C$ where

$$
C=\left(\begin{array}{ccccc}
I, & -\left(A_{11}-\lambda I\right)^{-1} A_{12}, & -\left(A_{11}-\lambda I\right)^{-1} A_{13}, & \cdots & -\left(A_{11}-\lambda I\right)^{-1} A_{1 n} \\
0 & I & 0 & \cdots & 0 \\
0 & 0 & I & \cdots & 0 \\
\vdots & & 0 & \cdots & I \\
0 & 0 & 0
\end{array}\right) .
$$

We see that $A^{(1)}$ is a matrix of the same type as $A$, and $\left|A^{(1)}\right|=|A-\lambda I|$, since $|C|=1$. If $B^{(1)}$ and $B^{(2)}$ are derived from $A^{(1)}$ and $A-\lambda I$ respectively in the same way that $B$ is from $A$, then $B^{(1)}=B^{(2)}$. But $A^{(1)}$ is such that $A_{11}^{(1)}=A_{11}-\lambda I$ and $A_{11}^{(1)}=0$, $(i \neq 1)$, so that $B^{(1)}=\left(A_{11}^{(1)}-\lambda I\right) D$ where $D$ is derived from the minor matrix of $A_{11}^{(1)}-\lambda I$ in the same way as $B$ from $A$, and $\left|A^{(1)}\right|=\left|A_{11}^{(1)}\right||D|$ since our proof by induction assumes that the theorem holds for $(n-1) \times(n-1)$ matrices of matrices. But $\left|B^{(2)}\right|=\left|B^{(1)}\right|=\left|A_{11}\right||D|=\left|A^{(1)}\right|=|A-\lambda I|$. Hence the theorem follows for infinite fields.

As all finite fields are Galois fields derived from the field of all rational functions, an identity of above type that holds in the latter field is also true for Galois fields.

UNIVERSITY OF WISCONSIN 